\title{
Research Progress of the Wild Medicinal Plant, Pinellia ternata
}

\author{
Xu Ding*, Quanhua Song, Wei Hu \\ Ankang Vocational and Technical College, Ankang 725000, Shaanxi Province, China \\ *Corresponding author: Xu Ding, ding1_1@163.com
}

\begin{abstract}
Based on literature reviews and analysis of research reports on Pinellia ternata found locally and abroad in recent years, this article summarizes and arranges them. The research on Pinellia ternata mainly focuses on its cultivation, tissue culture, and so on. There are only a few research on its active components and its regulation mechanism. The wild resources of Pinellia ternata are gradually decreasing, hence it is urgent to take effective measures to protect these wild resources as well as to establish germplasm resources bank and nursery. In order to meet the needs of the domestic market, it is necessary to investigate the distribution of wild Pinellia ternata resources, explore the best growing environment and conditions, artificially cultivate Pinellia ternata, as well as implement resource industrialization, sustainable development, and utilization.
\end{abstract}

Keywords: Pinellia ternata Thunb.; Traditional Chinese medicine resources; Identification of crude drugs; Active ingredient

Publication date: July 2021; Online publication: July 31, 2021

\section{Resources of Pinellia ternata}

Pinellia ternata (Thunb.) Breit. is derived from the dry tuber of Pinellia ternata (Thunb.) which is collected in the Chinese Pharmacopoeia. It has the effects of eliminating dampness, reducing phlegm, preventing nausea, relieve stuffiness and dissipate nodulation. ${ }^{[1]}$ Pinellia ternata is a common medicinal plant which is highly praised by physicians in past dynasties. It is not only used in traditional Chinese medicine prescriptions but also used as raw materials in various Chinese patent medicines.

Tubers of Pinellia ternata are spherical, 1-2 cm in diameter, with fibrous roots. It usually has 2 to 5 leaves, the petiole is 15 to $20 \mathrm{~cm}$ long, and base sheathing. There are bulbils of 3 to $5 \mathrm{~mm}$ in diameter at the middle or proximal part of the petiole and at the base of the leaf (tip of petiole). The bulbils germinate on the mother plant or after landing. The leaves of seedlings are ovate, heart-shaped to halberd-shaped, single, 2 to $3 \mathrm{~cm}$ long and 2 to $2.5 \mathrm{~cm}$ wide. The leaves of the old plant are 3-lobed, green in color with pale back, oblong-elliptic or lanceolate, sharp at both ends. The middle lobe is 3 to $10 \mathrm{~cm}$ long and 1 to 3 $\mathrm{cm}$ wide while lateral lobes are slightly shorter. Entire or with inconspicuous shallowly undulate rounded teeth, it has 8 to 10 pairs of lateral veins with dense veinlets reticulate and collecting veins 2 -circled. The peduncle is longer than the petiole, 25 to $30 \mathrm{~cm}$ long. With spathe green or greenish white, the tube is narrowly cylindrical, 1.5 to $2 \mathrm{~cm}$ long. Its eaves are oblong, green, sometimes with bluish purple margins, 4 to $5 \mathrm{~cm}$ long and $1.5 \mathrm{~cm}$ wide, obtuse or acute. They are fleshy inflorescence in which females are $2 \mathrm{~cm}$ long whereas males are 5 to $7 \mathrm{~mm}$ long by which they are $3 \mathrm{~mm}$ apart. Appendages are green to bluish purple, 6 to $10 \mathrm{~cm}$ long, erect, sometimes "s-curved." Berries are ovoid, yellowish green, with permanent stigma and style. The flowering period is from May to July and its fruits mature in August. It is widely distributed in China except in Inner Mongolia, Xinjiang, Qinghai, and Tibet. It grows in an altitude below 2500 meters and commonly found in grasslands, wastelands, corn fields, field edges, or in sparse forests. It is one of the weeds in dry land. It can also be found in North Korea and Japan. Tuber as both medicine and 
toxic can dry phlegm-dampness, treats cough, in addition to its anti-nausea and anti-vomiting properties. It can also be used in the treatment of acute mastitis as well as acute and chronic otitis media. On the other hand, it is used by veterinarians to treat the head and neck of animals. This species prefers warm and humid environments. It is a shade-tolerant species in which it can be planted in forests, between rows of fruit trees, or with other crops. It can be propagated by tubers, bulbils, or seeds. ${ }^{[2]}$

Li Ting, Li Min, and other researchers noted that the reserves of Pinellia ternata resources in China have significantly decreased and cultivation technologies are also lagging behind. The existing wild resources are in urgent need of protection. ${ }^{[3]}$ Wang Huadong and Wu Fansheng reported that the wild resources of Pinellia ternata in China are mainly distributed in Hubei, Sichuan, Anhui, and other provinces whereas in Gansu, Shaanxi, Chongqing, and other autonomous regions, there are different amounts of distribution. Only a few provinces or autonomous areas such as Inner Mongolia, Jilin, Heilongjiang, Xinjiang, Qinghai, and Tibet do not have any written information on the distribution of Pinellia ternata resources neither have never appeared in the market. ${ }^{[4]}$ In recent years, with the continuous increase in the market demand of Pinellia ternata, the decrease of its wild resources, and backward cultivation technologies, the incongruity between the three is becoming more serious. The destruction of the natural environment with excessive mining and the use of large amounts of pesticides result in a sharp reduction of wild resources which forms a vicious cycle with the resource prices. Zhou Jianli, Mu Erting, and other researchers noticed that the weed type Pinellia ternata has almost extinct in Huang Huai Hai Plain, MiddleLower Yangtze Plain, and Jianghan Plain. ${ }^{[5]}$ In addition to that, the genuine medicinal material of Pinellia ternata in Taizhou area of Jiangsu Province has been cultivated or is no longer produced.

Guo Qigui and Deng Yincai have investigated and studied the resources of traditional Chinese medicine in Ankang City. ${ }^{[6]}$ Through reference of historical data and market research of traditional Chinese medicine, they have selected genuine traditional Chinese medicine in Ankang City, a large number of dominant traditional Chinese medicine, as well as rare and precious traditional Chinese medicine. In recent years, the distribution area of wild Pinellia ternata decreased due to the return of farmlands to the forests as well as the abandoning of farmlands. With the increase of market demand and excessive mining driven by economic interests, wild resources have sharply decreased. Therefore, the protection of wild resources has become an urgent task.

\section{Active components of Pinellia ternata}

The active components of Pinellia ternata are complex. They include alkaloids, aspartic acid, glutamic acid, arginine, aminobutyric acid, Pinellia ternata protein, beta-sitosterol, glucoside, and alkapton. The contents of active ingredients directly reflect the quality of Pinellia ternata as a traditional Chinese medicine. Ji Wenlan and other researchers have determined the amino acid content in Pinellia ternata. ${ }^{[7]}$ The results showed that the total amino acid content of narrow leaf Pinellia ternata was higher than that of bamboo leaf Pinellia ternata. Moreover, the aspartic acid content in which it has certain antitussive and expectorant properties was also higher than that of bamboo leaf Pinellia ternata. These are used as important basis that the quality of narrow leaf Pinellia ternata is better than that of the bamboo leaf Pinellia ternata. Wei Shuhong and others showed that the total alkaloid content of narrow leaf Pinellia ternata was higher than that of the common Pinellia ternata by gravimetric method and acid dye colorimetry which also reflected that the quality of the narrow leaf Pinellia ternata was better than that of the common Pinellia ternata. ${ }^{\left[{ }^{8]}\right.}$ In regard to the total alkaloids content in Pinellia ternata, Baiquan and others stated that the content of total alkaloids in cultivated Pinellia ternata was different from that of wild Pinellia ternata. Even those from the same area have different total alkaloids content. However, the beta-sitosterol content in cultivated Pinellia ternata and wild Pinellia ternata is relatively consistent but there are differences in the betasitosterol content of Pinellia ternata from different habitats. The content of guanosine in cultivated Pinellia ternata was higher than that in wild Pinellia ternata. Yu Chao and other researchers believe that the alkaloid 
content of wild Pinellia ternata is generally higher than that of cultivated varieties. Zeng Jianhong also studied the changes of total alkaloid content in Pinellia ternata at different harvest times and concluded that the total alkaloid contents of Pinellia ternata at different harvest times were significantly different.

\section{Specific regions for the cultivation of Pinellia ternata}

The cultivation of Pinellia ternata is at certain regions. Henan, Hubei, Gansu, and other places have more cultivated Pinellia ternata whereas Sichuan, Anhui, Jiangsu, and other places mostly have wild Pinellia ternata. The propagation of Pinellia ternata can be achieved by tuber propagation, bulbil propagation, or seed propagation whereby currently, tuber propagation is more common. Pinellia ternata prefers warm environment however, it is afraid of high temperatures. $15^{\circ} \mathrm{C}$ to $25^{\circ} \mathrm{C}$ are the best temperatures for its growth. When the temperature exceeds $30^{\circ} \mathrm{C}$, its growth will be inhibited and above $35^{\circ} \mathrm{C}$, it will completely stop growing. Gao Shangfeng ${ }^{[9]}$ and other researchers found that the shading effect of crops was not obvious through the comparative test of crop shading with shading net, however shading with $80 \%$ light transmittance promoted the growth of the above-ground parts of Pinellia ternata and prevented abnormal seedling falling due to high temperature. In addition, the increase in yield was obvious with an average yield increase of $34.6 \%$. The changes of chemical constituents of Pinellia pedatisecta under different light intensities were studied. It was found that the contents of four nitrogen-containing compounds in Pinellia pedatisecta increased with $60.0 \%$ shading. Pinellia ternata prefers water and is afraid of drought yet when the water content is high, it is easy for seedlings to damp off and tubers to rot. Pinellia ternata is a widely distributed species which has strong adaptability to the environment. However, for the cultivation of Pinellia ternata to improve its yield, suitable cultivation conditions should be selected. The soil should be deep and fertile while drainage and irrigation should be convenient, and the best of all is the use of sandy soil.

Tissue culture method is also used in the propagation of Pinellia ternata. The best culture conditions of Pinellia ternata tissue seedlings are discussed in the following. Vermiculite and perlite can keep water and air which are needed for the rooting of Pinellia ternata, and the survival rate is $98.0 \%$. Pinellia ternata is photophobic so when there is strong light, shading would be the most suitable means of regulation. It does not only regulate photosynthesis, but also affects plant growth through the regulation of temperature and humidity. Temperature has a great influence on the survival rate of Pinellia ternata whereby the range of $16^{\circ} \mathrm{C}$ to $25^{\circ} \mathrm{C}$ is the most favorable for its survival and growth. During the growth period of Pinellia ternata, proper fertilization has a significant effect on its survival rate. A factory seedling system of Pinellia ternata has been established in terms of explants induction, plant growth regulator concentrations, light conditions, and subculture cycle experiments. For high-quality provenance of Pinellia ternata, tissue culture technology is able to obtain a large number of high-quality seedlings rapidly, hence this method has certain advantages. However, the cost of tissue culture is high in addition to difficult technical operations, so it is not suitable for small-scale cultivation of Pinellia ternata. Regenerated plantlets are obtained when callus is induced from dormant or just germinated tubers of Pinellia ternata. Other than that, when bulbils, small stems, leaves, petioles, and seeds of Pinellia ternata are used as explants for tissue culture, regenerated plants can also be obtained.

\section{Identification methods of Pinellia ternata}

The traditional identification methods of Pinellia ternata include source identification, character identification, microscopic identification, and physicochemical identification. Pinellia ternata is spherical but some are slightly oblique. Araceae is oblate, some of which have small oblate lateral buds. Water Pinellia ternata is conical, semicircular, or elliptic, and some of them are slightly pointed at the lower end. The cross section of Pinellia ternata is yellow, the cross section of water Pinellia ternata is white with 
many purple spots scattered over it. The lower end of Pinellia ternata is blunt, round, and smooth while the lower end of water Pinellia ternata is slightly sharp. Microscopically, there are less starch grains in Pinellia ternata powder but there are more in water Pinellia ternata powder. It is found that Pinellia ternata has a maximum absorption peak at $260 \mathrm{~nm}$ whereas the maximum absorption peak of water Pinellia ternata is at $273 \mathrm{~nm}$. On the other hand, the absorption peak of Arisaema is at $270 \mathrm{~nm}$ and the maximum absorption peak of Aconitum carmichaelii is at $256 \mathrm{~nm}$. Thin-layer chromatography (TLC) identification showed that Pinellia ternata and the reference substance showed the same color spots on the corresponding position but Arisaema yunnanense Buchet did not show the same color spots on the corresponding position with arginine as reference substance.

The fingerprints of Pinellia ternata are as follows. Electrochemical fingerprints of traditional Chinese medicine (TCM) are obtained by using the chemical oscillation technique to determine the variation of potential in the TCM system. Results have shown that the electrochemical fingerprints of Pinellia ternata and water Pinellia ternata are very similar in which the highest potential is the same. However, the induction time, oscillation life, and oscillation period of Pinellia ternata are less than those of the water Pinellia ternata. These results showed that the electrochemical fingerprints of Pinellia ternata are obviously different, hence they could be used to identify Pinellia ternata from the water Pinellia ternata. The high-performance liquid chromatography (HPLC) fingerprint of Pinellia ternata from Shandong Province has also been analyzed. The results showed that the similarity of Pinellia ternata collected from Shandong was above 0.9 which proved that the fingerprints of Pinellia ternata collected from Shandong are similar. The wild Pinellia ternata of Paeonia lactiflora leaf and bamboo leaf are used in clinics at Nanchong, Sichuan Province. Random amplified polymorphic DNA (RAPD) fingerprinting and polymorphism analysis have been used to identify them. The results showed that there are significant differences in the DNA fingerprints between the two but the differences in efficacies and compositions are not clear. The full-length cDNA of Pinellia ternata lectin has been cloned from inflorescences of Pinellia ternata using RACE-PCR. The $18 \mathrm{~S}$ rRNA nucleotide sequences of Pinellia ternata and its adulterants have been sequenced by PCR direct sequencing technology ${ }^{[10]}$.

\section{Conclusion}

Pinellia ternata is one of the most important pieces of traditional Chinese medicine and raw materials of Chinese patent medicine. It is one of the most frequently used herbs in classical prescriptions and it has definite curative effects. Although the wild distribution is very wide, due to the destruction of the ecological environment and large numbers of excavations in recent years, the wild resources of Pinellia ternata are gradually reducing, hence it is urgent to take effective measures in order to protect its wild resources. The establishment of germplasm resource bank and nursery are essential methods to protect the species diversity of Pinellia ternata. In order to meet the needs of the domestic market, it is necessary to investigate the distribution of wild Pinellia ternata resources, explore the best growing environment and conditions, artificially cultivate Pinellia ternata, as well as implement resource industrialization, sustainable development, and utilization. The active components of Pinellia ternata have been detected and analyzed. The active components in wild Pinellia ternata from different areas are compared to look for varieties and the producing areas with the highest content of medicinal ingredients.

\section{Funding}

Supported by Ankang Science and Technology Bureau, Shaanxi Province (2018ak03-11) and National Traditional Chinese Medicine Characteristic Technology Inheritor (t20194828003). 


\section{Disclosure statement}

The author declares no conflict of interest.

\section{References}

[1] 2015, State Pharmacopoeia Commission, Chinese Pharmacopoeia Volume 1, Chemical Industry Press, Beijing, 119.

[2] 1979, Flora of China. 13(2): 203.

[3] Li T, Li M, Jia JJ, et al., 2009, Investigation on Resources and Production Status of Pinellia ternata in China. Modern Chinese Medicine Research, 23(2): 11-3.

[4] Wang HD, Wu FS, 2012, Investigation on Pinellia ternata Resources in China. Anhui Agricultural Sciences, 40(1): 150-1.

[5] Mu ET, Zhou JL, 2013, Overview of Pharmacognosy of Pinellia ternata. Journal of Anhui University of TCM, 10(5): 91-3.

[6] Guo QG, Deng YC, 2007, Investigation and Research on TCM Resources in Ankang City. Journal of Ankang University, 19(2): 90-101.

[7] Ji WL, Yang QH, 1997, Determination of Amino Acid Content in Pinellia ternata of Different Germplasm, 8(6): 554.

[8] Wei SH, Peng ZS, 2003, Comparison of Total Alkaloids between Pinellia ternata and Pinellia ternata. Research and Practice of Modern Chinese Medicine, 34(12): 1133-5.

[9] Gao SF, Han JQ, 2006. Study on the Effect of Pinellia ternata Shading on Increasing Yield. Modern Chinese Medicine Research and Practice. 20 (3) : 23-25

[10] Zhang YL, Li GR, Wei YL, 2007, Research Progress of Pinellia ternata. Basic Science of Agriculture, 23:163-6. 